\title{
EMERGÊNCIA: FATORES QUE LEVAM A POPULAÇĀO INFANTIL A RECORRER A ESTE SERVIÇO *
}

Ninon Girardon da Rosa Martins **

Lisiane Manganeli Girardi Paskulin ***

\begin{abstract}
RESUMO - A deficiência dos serviços básicos de saúde vem determinando um aumento na demanda aos serviços de atendimento terciário. Essa situação sobrecarrega as unidades de emergência, mobiliza desnecessariamente recursos humanos e materiais especializados, reforça a desvalorização da ação preventiva e soluciona paliativamente os problemas da população. Este problema reflete-se na procura do Serviço de Emergência do Hospital de Clínicas de Porto Alegre, cujo nível de assistência aos problemas de saúde da criança procura-se medir. Para isto utilizou-se instrumento próprio e entrevista que permitiram levantar os problemas de saúde e os motivos de procura ao serviço. Constata-se que a maioria dos atendimentos necessita ser trabalhado no primeiro nível de assistência e analisa-se a política de saúde e o papel do enfermeiro neste contexto.
\end{abstract}

\begin{abstract}
The absence of a health service has been determining an increasing demand over the tertiary system. The emergency services are overloaded, human and material resources are unnecessarily mobilized, prevention action is devaluated and the population's problems are only partially solved in this situation. This problem is reflected in the search of Hospital de Clínicas' emergency unit whose assistence level to child health problems is measured in this research. For that, an interview and an instrument are utilized in order to obtain data about child health problems and the motives for searching the service. It is verified that the most part of attendances could be resolved at the 1 st assistance level. Finally, health politics and nurses' role are analysed facing this context.
\end{abstract}

\section{INTRODUÇĀO}

A política de saúde atual parece estar determinando um estrangulamento dos recursos existentes. Constata-se, na prática, que não há uma preocupação com os aspectos de promoção, manutenção e educação para a saúde. Observa-se, ainda, que a prioridade está voltada para o aspecto curativo em detrimento do preventivo. Em função disso, verifica-se que os recursos financeiros estão sendo alocados ao setor terciário.

Atuando em uma unidade de emergência de um hospital universitário, pôde-se constatar a deficiência dos serviços de saúde através do aumento constante e desenfreado de uma utilização pelo público, gerando uma superpopulação a ser atendida.

Essa situação já começa a ser identificada no momento em que o enfermeiro realiza a triagem, quando avalia os pacientes e determina as propriedades no atendimento. Dessa forma, observa-se que o motivo que leva os pacientes a procurarem o serviço de emergência, não necessitaria de um tratamento em um setor terciário, mas poderia ser manejado em níveis primário e secundário. A especialidade é necessária desde que planejada e bem utilizada. O que deve ser repensado é o atendimento em nivel primário de assistência, não como um serviço simplista, mas simplificado e que atenda às reais necessidades da população nas suas articulações, com os niveis secundário e terciário.

A escolha desse tema partiu de um questionamento sobre os fatores que estariam levando os responsáveis pela população infantil à procu-

\footnotetext{
* Prêmio Isaura Barbosa Lima - 2ㅇ Lugar - $41^{\circ}$ - Congresso Brasileiro de Enfermagem - Florianópolis-SC.

** Enfermeira da Unidade de Emergência do Hospital das Clínicas de Porto Alegre. Licenciada em Enfermagem.

*** Enfermeira da Unidade de Emergência do Hospital das Clínicas de Porto Alegre. Licenciada em Enfermagem.
} 
ra direta de serviços de alta complexidade, quando se sabe que a prioridade da política brasileira de saúde é a prestação da assistência materno-infantil e que esta deveria estar presente em todos os niveis de atendimento.

O trabalho diário junto à clientela que procura a unidade de emrgência, faz com que se conheça empiricamente o perfil da demanda. É intenção das autoras registrar, nesse estudo dados referentes a essa prática, para gerar subsídios aos técnicos de saúde no conhecimento da realidade com a qual trabalham, e servir, também, de apoio técnico à implantação do Sistema Único de Saúde.

\section{Objetivos}

- Verificar os problemas de saúde das crianças que são atendidas no Serviço de Emergência. rio.

- Identificar o nível de assistência necessá-

- Levantar os motivos que levam a população a procurar diretamente um serviço terciário.

- Refletir sobre a política de saúde brasileira e o papel do profissional de saúde dentro do sistema.

\section{METODOLOGIA}

O estudo foi realizado no Serviço de Emergência do Hospital de Clínicas de Porto Alegre, tendo como amostra 347 crianças na faixa etária de zero a quatorze anos incompletos, no perfodo de maio a junho do ano de 1989.

Utilizou-se um instrumento com a intenção de verificar se, o problema de saúde dos pacientes necessitaria ser trabalhado em nfvel de um setor terciário. Os dados levantados no instrumento foram: a idade das crianças, o motivo da consulta, a hipótese diagnóstica e o tratamento respectivo. Esses números foram coletados com base nos boletins de atendimento das crianças consultadas. Considerou-se as vinte e quatro horas de dois dias de atendimento, divididos em três intervalos: das $0 \mathrm{~h}$ às $7 \mathrm{~h}$; das $7 \mathrm{~h}$ às $18 \mathrm{~h}$; das $18 \mathrm{~h}$ às $24 \mathrm{~h}$. Foram escolhidas duas quintas-feiras, em semanas alternadas, em função de ser um dia que retrata a média de atendimento na Unidade de Emergência.

Sabe-se que no mês de maio foram atendiddas 5.231 crianças; semanalmente 1.308 crianças e diariamente em torno de 169 crianças. Porém, segunda-feira é um dia em que aumenta consideralmente o número de consultas e no fim-se-semana elas se reduzem sensivelmente.

É importante ressaltar que, de 400 pacientes que procuraram a emergência nestes dois dias, avaliaram-se 347 boletins de atendimento, pois
52 foram perdidos ou houve desistência de consultas.

Entrevistaram-se as mães dos pacientes atendidos no segundo dia do levantamento, no momento da triagem desses pacientes, quanto ao motivo que as levou a recorrer a uma unidade de emergência como alternativa de atendimento. Foram entrevistadas 146 mães das 178 consultas realizadas neste dia.

A partir dos dados obtidos, realizou-se ainda a classsificação do nível de atendimento (primário, secundário ou terciário) que cada criança necessitaria, tendo por base o motivo da consulta, a hipótese diagnóstica e o tratamento necessário. Essa classificação foi realizada com base no modelo de atendimento apresentado a seguir:

Em 1987 foi criado o Sistema Único Descentralizado de Saúde com o objetivo de municipalizar e equalizar as ações dessa área, continuando o processo de unificação do sistema das Ações Integradas de Saúde. Com a promulgação da Constituição em 1988, toma forma o Sistema Único de Saúde e, atualmente, discute-se a definição da lei orgânica desse sistema.

O Instituto Nacional da Previdência e Assistência Social, em 1988, propôs um modelo, a partir desta política de saúde, com os serviços localizados dentro de Distritos Sanitários. O Distrito Sanitário é considerado "uma base territorial definida geográfica e demograficamente dispondo de rede de serviços de saúde com perfil tecnológico adequado às características epidemiológicas daquela população". Dentro do Discrito Sanitário devem existir três níveis de assistência: no $1^{\circ}$ nf́vel seriam realizadas ações de cuidado individual e coletivo, em relação permanente com a população; no 2 - nivel haveria a prestação de serviços de apoio diagnóstico e terapêutico; o 30 nível prestaria atendimento em situações emergenciais e internações. $\mathrm{O}$ modelo enfatiza a importância do $1 \%$ nível como porta de entrada da população.

Determinou tamberm, as atividades a serem desenvolvidas em cada nível:

- 10 nfvel de assistência: assistência clínica geral a crianças e adultos, imunizações, assistência odontológica, coleta de material para exame diagnóstico, dispensa de medicamentos, atividades em educação e nutrição para a saúde, notificação compulsória das doenças e vigilância epidemiológica, atividades de documentação assistencial e visitação domiciliar;

- 20 nfvel de assistência: assistência clínica especializada, realização de exames diagnósticos, realização de procedimentos terapêuticos especializados, dispensa de medicamentos, notificação compulsória de doenças, atividade de documentação assistencial e orientação técnica das unidades de $1 \%$ nível;

- 39 nfvel de assistência: assistência clf- 
nica a pacientes internados, atendimento ambulatorial especializado, atendimento de emergência nas $24 \mathrm{~h} / \mathrm{dia}$, atendimento em regime hospital-dia, realização de procedimentos diagnósticos e terapêuticos, dispensa de medicamentos, notificação compulsória de doenças.

\section{APRESENTAÇĀO E DISCUSSĀO DOS RESULTADOS}

A média de idade da amostra em questão é de 3,689 , variando de zero a quatorze anos incompletos.

Tabela 1 - Distribuição da faixa etária da amostra Hospital de Clínicas de Porto Alegre, maio e junho de 1989

\begin{tabular}{|c|c|c|c|c|c|}
\hline Faixa & Etárıa & $1^{2}$ dia & $2^{2}$ dia & $f$ & fr $(\%)$ \\
\hline $\mathrm{RN}$ & $-1 a$ & 3 & 13 & 16 & 4,61 \\
\hline $1 \mathrm{a}$ & $-2 a$ & 40 & 35 & 75 & 21,62 \\
\hline $2 a$ & $-4 a$ & 29 & 34 & 63 & 18,16 \\
\hline $4 a$ & - $6 a$ & 40 & 34 & 74 & 21,32 \\
\hline $6 a$ & - & 21 & 20 & 41 & 11,81 \\
\hline od & - 10a & 25 & 29 & 54 & 15,56 \\
\hline 10 & - & 11 & 13 & 24 & 6,92 \\
\hline Total & & 169 & 178 & 347 & 100,00 \\
\hline
\end{tabular}

Fonte: Boletins de atendimento do Serviço de Emergéncia

Observa-se que a faixa etária que procurou atendimento na unidade de emergência concenra-se, principalmente, no período pós-neonatal ao pré-escolar. Essa faixa etária representa a prioridade do atendimento da população infantil brasileira, pois segundo o Ministério da Saúde, o objetivo mais amplo do governo, na assistên- cia integral à saúde da criança, é a redução da morbimortalidade na faixa do zero aos cinco anos de idade.

Estes resultados vêm confirmar que os serviços básicos de saúde não estão absorvendo justamente a demanda empreendida na faixa de idade priorizada pelo governo.

Tabela 2 - Distribuição das hipóteses diagnósticas encontradas na amostra

\begin{tabular}{|c|c|c|}
\hline Hipotese diagnostica & $f$ & fr $(\%)$ \\
\hline Problema respi rató rio & 198 & 50,50 \\
\hline Otite média aguda & 65 & 16,58 \\
\hline Problemas gast rointestinais & 43 & 10,92 \\
\hline Febre & 18 & 4,59 \\
\hline Virose & 13 & 3,34 \\
\hline Impetigo & 8 & 2,06 \\
\hline Esto matite herpética & 7 & 1,78 \\
\hline Problema dermatológico & 5 & 1,29 \\
\hline Problema neu rológico & 5 & 1,27 \\
\hline Doenças infecto-contagiosas & 4 & 1,04 \\
\hline Problemas u rinários & 4 & 1,02 \\
\hline Recém-nascidos sem alteraçōes & 4 & 1,02 \\
\hline Outros & 18 & 4,59 \\
\hline Total & 392 & 100,00 \\
\hline
\end{tabular}

Fonte: Boletins de atendimento do Serviço de Emergéncia

A partir dos dados obtidos em relação aos problemas de saúde apresentados pelas crianças, identifica-se altíssima freqüência dos problemas do trato respiratório que se caracterizam em doenças típicas do Rio Grande do Sul.

Conforme a Secretaria da Saúde e a CICDISETEC/CIS ${ }^{2}$, os principais agravos que compõem o quadro de mortalidade infantil, no Rio Grande do Sul, são as infecções respiratórias agudas.

Seguindo-se aos problemas respiratórios encontram-se as otites médias agudas e os problemas do trato gastro-intestinal (gastroenterite aguda). Este último com menor incidência, em função de a estação climática do ano favorecer o aparecimento dos problemas respiratórios, em detrimento da desidratação e da gastroenterite aguda.

Dados que vêm reforçar a necessidade do desenvolvimento dos serviços básicos de saúde, é o tratamento simplificado (orientação, tratamento sintomático e outros) aplicados a grande parte dos problemas respiratórios e gastrointestinais. A conduta de orientação aos pais também ocorre quando do diagnóstico de doenças infecto-contagiosas e do atendimento do recém-nascido sem alterações.

O número de hipóteses diagnósticas levantadas foi maior do que o número total de atendimentos, porque algumas crianças apresentavam mais de um problema de saúde concomitantemente. 


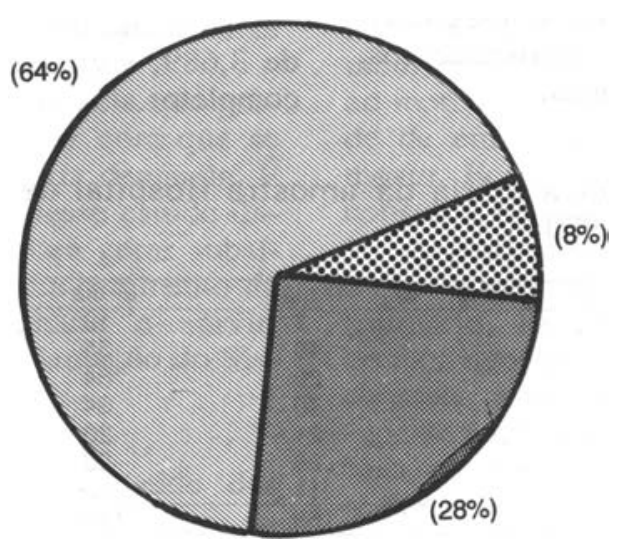

Primário

Secundário Terciário

Observação: Os valores apresentados no gráfico estâo arrendodados

Observa-se que na classificação dos níveis de atendimento a maior freqüência se concentra no nível primário $(64,27 \%)$, seguindo-se o nível secundário $(27,95 \%)$ e, em menor número, no nível terciário $(7,78 \%)$.

É importante registrar que de um total de 347 atendimentos, $82(23,63 \%)$ foram no periodo das 0 (zero) às $7 \mathrm{~h}$ e das 18 às $24 \mathrm{~h}$. Nesse horário, normalmente os serviços de atendimen- to em níveis primário e secundário estão fechados; porém, verifica-se que a maioria dos motivos das consultas não necessitariam recorrer a uma unidade de emergência nesse horário. Do número total, somente quatro $(4,88 \%)$ pacientes precisariam de atendimento de emergência.

Classificaram-se, também, os niveis de atendimento, em relação aos problemas respiratórios encontrados na população-alvo:

\section{Gráfico 2 - Problemas respiratórios apresentados pela amostra segundo os níveis de atendimento}
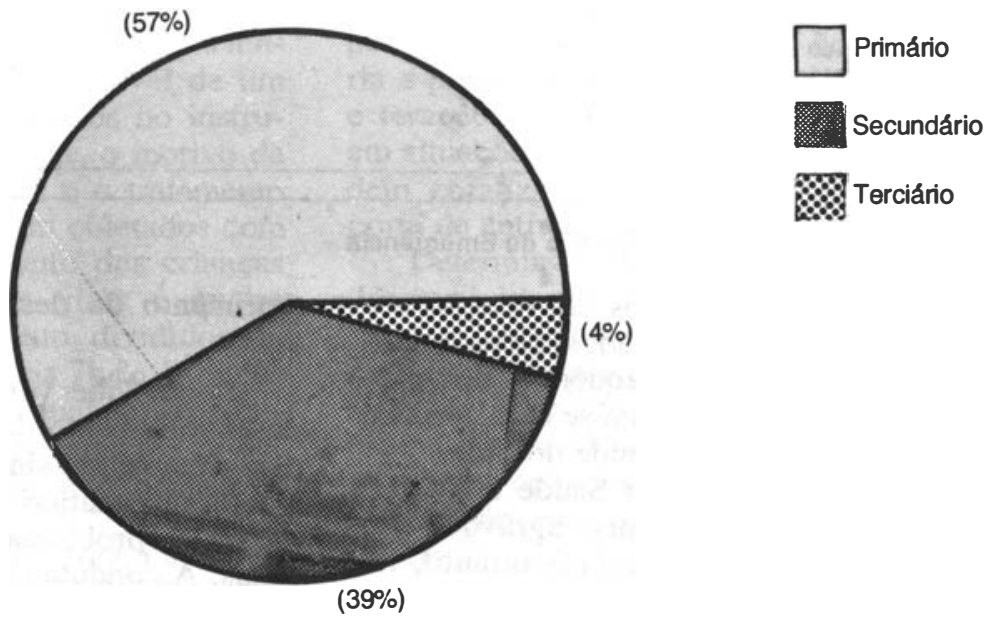

$(4 \%)$

Observaçáo: Os valores apresentados no gráfico estáo arrendodados

Verifica-se que, de um total de 198 problemas respiratórios, a maior freqüência do nível primário permanece $(57,06 \%)$, logo após, en nível secundário $(38,89 \%)$ e, em menor concentração, em nível terciário $(4,04 \%)$. 


\section{- Demanda dos serviços especialidados}

Surge o questionamento sobre o porquê da elevada demanda aos serviços especializados.

Em entrevista realizada com as mães dos pacientes que procuraram o serviço de emergência obtiveram-se as seguintes respostas:

\section{Tabela 3 - Motivos que levaram a amostra estudada a procurar o Serviço de Emergência do HCPA}

\begin{tabular}{|c|c|c|}
\hline Motivos & f & fr $(\%)$ \\
\hline Sempre consulta na emergência & 21 & 13,29 \\
\hline Problema de atendimento no posto de saúde * & 18 & 11,39 \\
\hline Preferéncia pelo atendimento no HCPA & 16 & 10,13 \\
\hline Acha que é o local mais p róximo & 16 & 10,13 \\
\hline Encaminhado & 14 & 8,86 \\
\hline Rapidez no atendimento & 13 & 8,23 \\
\hline Porque acha que é uma emergência & 12 & 7,59 \\
\hline \multicolumn{3}{|l|}{ Consultou em outros locais mas a criançăo náo } \\
\hline obteve melhora & 10 & 6,33 \\
\hline Paciente do ambulató rio do HCPA & 10 & 6,33 \\
\hline Nasceu no HCPA & 8 & 5,07 \\
\hline Os pais são funcionários do HCPA & 5 & 3,17 \\
\hline Desconhece outros locais de atendimento & 3 & 1,89 \\
\hline Pediatra não estava atendendo & 2 & 1,26 \\
\hline Greve nos hospitais do INAMPS & 2 & 1,26 \\
\hline Outros & 8 & 5,07 \\
\hline Total & 158 & 100,00 \\
\hline
\end{tabular}

O número de motivos de consulta é maior do que o número total de mães entrevistadas, porque algumas colocavam mais de um motivo concomitantemente.

Os dados demonstraram que, com grande freqüência, a população utiliza o serviço de emergência como o ambulatório, um serviço de pronto atendimento. As respostas que reforçam essa afirmação são: "sempre consulta na emergência, o atendimento ê mais rápido, não É necessário marcar hora, pais funcionários do hospital, pediatria nāo estava atendendo". Dentro desse aspecto, verifica-se ainda que a emergência funciona como "válvula de escape", pois assume o excesso da demanda e a falta de estrutura dos serviços básicos de saúde, levando os pacientes a procurarem diretamente um serviço especializado.

Outro fator a destacar é que, os próprios profissionais de saúde, sabendo da problemática, encaminham seus clientes que, a rigor, não careceriam de um atendimento em nivel terciário, para as emergências.

As respostas de preferência pelo atendimento na instituição e, a procura da mesma como alternativa de solução de problemas não resolvidos em outros locais, sugerem que a confiança da população nos serviços de saúde é diretamente proporcional à complexidade dos mesmos.

Essas situações ratificam o desconhecimento da população em relação à dinâmica do atendimento de saúde e aumentam a sobrecarga dos serviços de alta complexidade.
Entretanto, é compreensivel esse desconhecimento quando analisa-se a história da política de saúde brasileira. Já em 1980, MAGALHÂES ${ }^{5}$, em sua reflexão sobre essa política, afirma que nos últimos 50 anos houve mais um esforço em estabelecê-la, depois uma luta para substitui-la por outra, que nunca pode ser realizada. Verifica-se que a história se repete na decada de 80 , pois existiu a tentativa de implantação de planos como as Ações Integradas de Saúde, o Sistema Único Descentralizado de Saúde e o Sistema Único de Saúde, sem uma real concretização e implementação de uma política de saúde definida.

A partir disso, verifica-se que o atendimento em nivel preventivo continua sendo desvalorizado e que muitas vezes os problemas são trabalhados em um serviço de alta complexidade, inadequadamente. Dessa maneira, têm-se gastos mais elevados, mobilização desnecessária de recursos humanos e materiais e, solução paliativa dos problemas, sem considerar a prevenção e a continuidade no atendimento.

LANDMAN ${ }^{4}$, comparando parses desenvolvidos e subdesenvolvidos, mostra que o aumento dos gastos em medicina curativa reduzem a mortalidade infantil em indices bem inferiores aos gastos equivalentes em saúde pública.

MANCIAUX $^{6}$, em sua análise sobre o atendimento à saúde da criança, representa, através de um gráfico, a inter-relação entre a necessidade, o pedido e o serviço e, afirma ser esta congruência fraca. A necessidade traduz a exigência de um serviço de atendimento em nf- 
vel terciário, a solicitação significa o pedido expresso pela população de serviços primários e secundários e, o serviço simboliza o preparo técnico e material da instituição para atender às necessidades constatadas.

- Inter-relação entre a necessidade, a solicitação e o serviço.

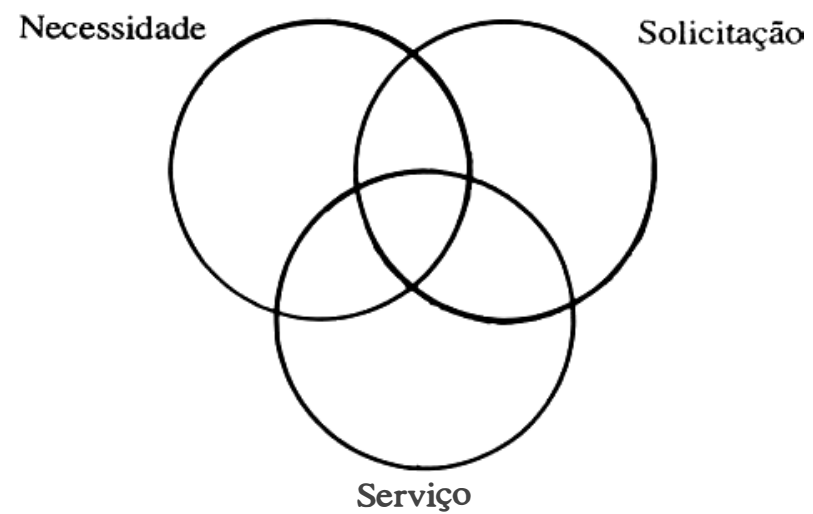

$\mathrm{O}$ autor reforça, ainda, que as ações que devem ser desenvolvidas para aumentar esta congruência, seriam um melhor conhecimento da situação, a educação em saúde e o desenvolvimento dos serviços básicos de saúde.

A partir da constatação da inexistência de uma política de saúde definida, da falta de assistência integral e contínua à criança, da desinformação da população na utilização dos serviços de saúde, é necessário que se reflita sobre o papel do profissional de saúde dentro deste contexto.

O primeiro passo é o conhecimento da realidade com a qual se trabalha.

Segundo REZENDE ${ }^{7}$ a postura alienada dos profissionais de saúde, face às realidades regionais, pode conduzir a difusão de medidas inadequadas à regionalidade dos Clientes, gerando um confrontamento de valores.

FREIRE $^{3}$ coloca em sua análise sobre o compromisso do profissional que procura a mudança da realidade que está "molhado e ensopado" da situação concreta, terá condições de se enganjar no mundo em que vive e, dessa maneira, comprometer-se com a sociedade. Reforça, ainda, que no processo de mudança não há saber $\mathrm{e}$ ignorância absoluta $\mathrm{e}$ não pode haver passividade e imitação.

\section{CONCLUSĀO}

Nesse estudo pode-se constatar que está ocorrendo uma inversão no sistema de atendi- mento à população. $\mathrm{Na}$ proposta governamental a "porta-de-entrada" da procura aos serviços deve ser em nível primário porém, o processo está ocorrendo de maneira oposta. Os dados levantados indicam que o estrangulamento do serviço de emergência se deve à desestruturação dos serviços básicos de saúde. A população, então fica sem alternativa para a solução de seus problemas. Procura diretamente os serviços especializados, porque os de atendimento em níveis primário e secundário não são valorizados, pois não possuem recursos adequados que atinjam um grau de resolutividade.

A polftica de saúde se retrata historicamente pela supervalorização do enfoque curativo. Tem havido uma seqüência de propostas que visam a qualificação do nível de prevenção; entretanto, a viabilização destas não está na modificação constante do modelo, mas depende da redefinição de valores e interesses do grupo que as determina.

É motivo de preocupação o fato de a clientela ser assistida somente na fase agura do processo de doença, sem uma investigação, acompanhamento evolutivo e muitas vezes como solução momentânea.

A abordagem desse tema tornou oportuna a confirmação do conhecimento empírico da realidade. A partir disso, a proposta é divulgar as constatações, proporcionando aos técnicos de saúde, compartilharem dessa experiência, num processo de reflexão e conscientização, que favoreça a integração do sistema de saúde. 


\section{REFERÊNCIAS BIBLIOGRÁFICAS}

1 BRASIL. Ministério da Previdência e Assistência Social. Aperfeiçoamento e humanização das ações assistenciais. Brasília, 1988.

2 CICDI-SE TEC/CIS-RS. Proposta de atendimento interinstitucional à criança. Revista do HCPA. Porto Alegre: 7:104-7, 1987.

3 FREIRE, Paulo. Educação e Mudança. Riode Janeiro: Paz e Terra, $1979.79 \mathrm{p}$.

4 LANDMAN, J. Evitando a Saúde \& Promovendo a Doença. 3. ed., Rio de Janeiro: Achiamé, 1982. 187p.

5 MAGALHĀES, M. A política de saúde pública no Brasil nos últimos 50 anos, 2. ed., In: CÂMARA DOS DE-
PLi TADOS. I Simpósio sobre Política Nacional de Saúde. 1980.

6 MANCIAUX, M. Saúde da mãe e da criança: problemas do futuro, elementos de solução. In: CENTRE D'ÉTUDES ET DE RECHERCHES MARXIS TES. Saúde $e$ Educação de Infância. Lisboa: Editorial Estampa, 1978. 299p.

7 REZENDE, A. Aspectos políticos e ideológicos das demandas e ofertas de atenção de saúde. Revista Gaúcha de Enfermagem. Porto Alegre, 5 (1): 65-82, 1984.

8 RS.SSMA. Estatisticas de Saúde: mortalidade 1985. Porto Alegre, 1986. 78p. 